\title{
Thermophysical Properties of Thin Fibers via Photothermal Quantum Dot Fluorescence Spectral Shape-based Thermometry
}

Troy Munro

Mechanical Engineering Department, Brigham Young University, troy.munro@byu.edu

Liwang Liu

Department of Physics and Astronomy, KU Leuven

Heng Ban

Department of Mechanical and Aerospace Engineering, Utah State University

Christ Glorieux

Department of Physics and Astronomy, KU Leuven

Follow this and additional works at: https://scholarsarchive.byu.edu/facpub

Part of the Mechanical Engineering Commons

\section{Original Publication Citation}

Munro, T., Liu, L., Ban, H., and Glorieux, C., "Thermophysical Properties of Thin Fibers via Photothermal Quantum Dot Fluorescence Spectral Shape-based Thermometry," International Journal of Heat and Mass Transfer, 112, pp. 1090-1097, 2017.

\section{BYU ScholarsArchive Citation}

Munro, Troy; Liu, Liwang; Ban, Heng; and Glorieux, Christ, "Thermophysical Properties of Thin Fibers via Photothermal Quantum Dot Fluorescence Spectral Shape-based Thermometry" (2017). Faculty Publications. 1874.

https://scholarsarchive.byu.edu/facpub/1874

This Peer-Reviewed Article is brought to you for free and open access by BYU ScholarsArchive. It has been accepted for inclusion in Faculty Publications by an authorized administrator of BYU ScholarsArchive. For more information, please contact ellen_amatangelo@byu.edu. 


\title{
Thermophysical Properties of Thin Fibers via Photothermal Quantum Dot Fluorescence Spectral Shape-based Thermometry
}

\author{
Troy Munro ${ }^{\mathrm{a}, \mathrm{b}, \mathrm{c}, 1, *}$, Liwang $\mathrm{Liu}^{\mathrm{c}}$, Heng Ban $^{\mathrm{b}}$, Christ Glorieux $^{\mathrm{c}}$ \\ ${ }^{a}$ Department of Mechanical Engineering, Brigham Young University, 435 Crabtree Building, Provo, UT 84602, USA \\ ${ }^{b}$ Department of Mechanical and Aerospace Engineering, Utah State University, 4130 Old Main Hill, Logan, UT 84322, USA \\ ${ }^{c}$ Department of Physics and Astronomy, KU Leuven, Celestijnenlaan 200D, B-3001 Heverlee, Belgium
}

\begin{abstract}
To improve predictions of composite behavior under thermal loads, there is a need to measure the axial thermophysical properties of thin fibers. Current methods to accomplish this have prohibitively long lead times due to extensive sample preparation. This work details the use of quantum dots thermomarkers to measure the surface temperature of thin fibers in a non-contact manner and determine the fibers' thermal diffusivity. Neural networks are trained on extracting the temperature of a sample from fluorescence spectra in calibrated, steady-state conditions, based on different spectral features such as peak intensity and peak wavelength. The trained neural networks are then used to reconstruct the evolution of the surface temperature in transient heating experiments. In order to determine the thermal properties of a thin fiber, modulated laser heating is applied and an FFT-based method is used to extract the phase and amplitude response of the temperature field at the modulation frequency. The spatiotemporal dependence of the fluorescence signal, obtained by scanning the distance between the excitation and detection laser spots and varying the frequency response due to an axial scan and a frequency scan, is then curve-fit to the resulting decay curves by a photothermal model in order to determine the thermal diffusivity of the fiber. The measured thermal diffusivity $\left(3.3 \pm 0.8 \times 10^{-7} \mathrm{~m}^{2} \mathrm{~s}^{-1}\right)$ of a synthetic spider silk fiber by the current method has similar properties to other synthetic silk fibers, and demonstrates the ability of the current method to more rapidly measure thermophysical properties of thin fibers.
\end{abstract}

Keywords: photothermal, quantum dots, thermometry, FFT, neural networks, thermal diffusivity

\section{Introduction}

Quantum dots with significantly temperaturedependent fluorescence properties have been considered for use as nano-sized temperature probes [1, because of their increased stability over organic dyes [2]. It should be mentioned that their fluorescence intensity can still be decreased when in contact with oxygen [3]. However, the use of quantum dots for temperature sensing remains attractive despite these and other limitations due to their small size [4, and their applicability in a variety of applications [5]. Quantum dots are nanometer-sized semiconducting crystals, whose emission spectrum in general and fluorescence peak wavelength in particular are a function of their size. CdSe/ZnS quantum dots represent one class of multiple probes that have been considered for nanothermometry at the cellular level [6], where improved temperature resolution is required [7, 8, 4. They (and other fluorophores) have been used for thermal characterization of the solution they are

\footnotetext{
${ }^{*}$ Corresponding author

Email address: troy.munro@byu.edu (Troy Munro)

${ }^{1}$ Permanent address - Brigham Young University, 435 Crabtree Building, Provo, UT 84602, USA
}

suspended in [9, 10, for spectroscopic investigation of materials via the thermal lens and optical absorption methods 9 , and as contrast agents in photoacoustic and photothermal microscopy [11. Their contribution to the effective thermal diffusivity of the system of interest is negligible [9, which is necessary for thermal characterization of materials. However, the temperature resolution with state-of-the-art quantum dots is on the order of 1 K[4] and methods to improve this accuracy are desired. Previous work [10, 12] has demonstrated the potential for neural networks to improve this accuracy.

The accuracy of quantum dot thermometry can also benefit from the use of frequency domain-based methods. Many fluorescence spectroscopy measurements in the frequency domain have been focused on lifetime-based measurements 13, with the emphasis being on understanding the decay kinetics of chemical systems. These frequency domain lifetime measurement were coupled with a fast fourier transform (FFT) and intensity-modulated light source to induce fluorescence in a fiber optic-based temperature sensor [14, thereby improving the detected signal and aiding in fitting the lifetime decay as a function of temperature. However, for frequency domain modulated heating experiments (such as lock-in IR thermometry [15] or modulated optical reflectance [16]), the probe beam is 
usually continuously illuminating and the pump beam is modulated. The current study seeks to use quantum dot fluorescence thermometry as a method to probe the modulated surface temperature of fibers. Photoluminescence intensities in the time domain via spectrometer rather than lifetime measurements are investigated. This also motivates the investigation of fluorescent probes, rather than phosphorescent ones, because the lifetimes of typical phosphorescent probes are longer than desirable for typical frequencies used in photothermal methods [17.

There is the possibility to improve the signal-to-noise ratio of the FFT signal by improving the accuracy of the reconstructed temperature. Neural networks have successfully been used to reconstruct the surface signal for photothermal radiometric experiments [18. They have the potential to improve the temperature accuracy of the quantum-dot shape-based thermometry. The current study seeks to expand shape-based, neural network reconstructed, time-domain fluorescence thermometry [10, 12] with quantum dots. The reconstructed temperature evolution is then mapped into the frequency domain to determine the decay and phase delay of the thermal wave along a thin fiber caused by intensity-modulated laser heating for the purpose of determining thermal diffusivity.

Accurate measurements of the thermal properties of fibers are of interest, because of large uncertainties in the measured property for spider silk [19, 20, 21] and polyethylene nanofibers [22, which are induced by thermal contact resistance 23, modeling biases 24, and spatial resolution 25. Additionally, axial heat conduction (and to a lesser extent, thermal diffusivity $\alpha=k / \rho c_{p}$ ) and Youngs modulus are properties of interest because they are the most sensitive characteristics to measure chain extension and continuity in polymers [26]. Hence, a non-contact thermometry based method is investigated for measuring the thermal diffusivity of a thin fiber.

In this work, a neural network approach is used for extracting temperature information from the fluorescence spectrum of inorganic fluorophores, both in time- and frequency-domain. An FFT-based method is elaborated upon in order to perform this frequency domain mapping operation and obtain the needed amplitude and phase at the modulation frequency. Laser heating experiments and simulations are investigated to determine the feasibility of this approach to measure the thermal diffusivity of thin fibers.

\section{Experimental Setup}

The experimental setup consisted of a pump-probe laser system with the sample fiber mounted on a sample holder capable of measuring and controlling its temperature (Figure 1). Quantum dots (Lumidot ${ }^{\mathrm{TM}} 640 \mathrm{~nm}$ peak wavelength, CdSe/ZnS from Sigma Aldrich) deposited on the surface of the fiber were illuminated with the probe beam (532 nm Coherent Compass CW laser) at a spot size of $30 \mu \mathrm{m}$. The resulting fluorescent emission was collected by an Olympus microscope objective (10X,0.25 NA) into the fiber optic of an Ocean Optics USB4000 spectrometer, after having passed though band pass filters to remove the probe light. After passing through an optical chopper, the pump laser (a $1064 \mathrm{~nm}$ Coherent Vector) was focused onto the fiber with the top half of a bifurcated plano-convex lens mounted on a position scanning stage, with a resulting spot size of $90 \mu \mathrm{m}$. The laser beam contained some residual green light, which was filtered out prior to arriving at the sample, and the reflected light from the filter was focused onto the spectrometer to provide a reference for the intensity-modulation of the laser light for photothermally generated temperature variations.

The longer wavelength of the pump beam was selected to not induce fluorescence of the quantum dots. The absorption of the quantum dots at IR wavelengths is insignificant, so that the pump beam did not interfere with the emitted fluoescence spectra. Focusing of the lasers by half lenses allowed independent motion of the pump laser from the probe laser, as well as maintaining the same focal plane for both collimated laser beams.

The sample holder consisted of an aluminum plate with four heating resistors that provided control of the sample temperature from a Labview-controlled PID control routine. The fiber was placed in a slit on the plate to allow optical access for front and rear illumination. Temperature was measured by both an Omega F3141 PT1000 RTD (measured by an HP-34401 via GPIB control, with an uncertainty of $0.065 \mathrm{~K}$ ) and an Omega type $\mathrm{T}$ thermocouple (welded in-house, measured by an HP-34970A via GPIB with an uncertainty of $0.1 \mathrm{~K}$ ). Samples were placed inside a Janis cryostat to provide optical access and high vacuum $\left(10^{-5} \mathrm{mTorr}\right)$. The significantly decreased vacuum pressure insured the absence of convective contributions to the heat transfer around the fiber [27].

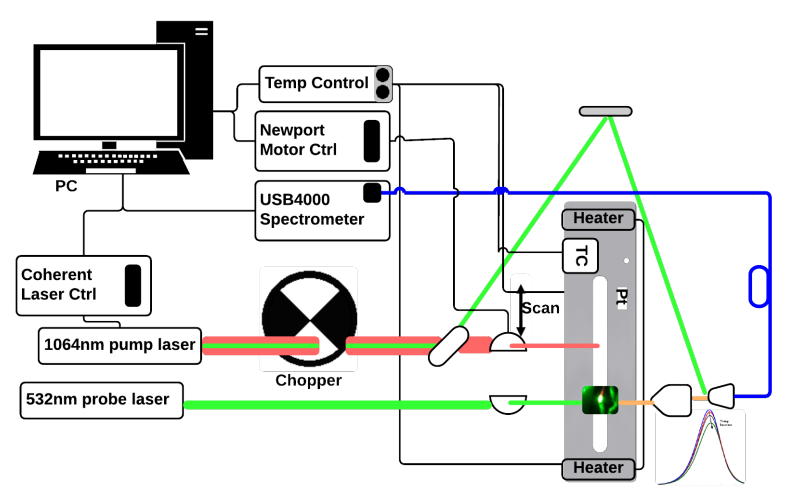

Figure 1: Experimental setup for quantum dot fluorescence measurement.

In order to relate the fluorescence spectra to the fiber surface temperature, a calibration is needed. The calibration process of the experiment began by stabilization of the fiber at $\pm 0.015{ }^{\circ} \mathrm{C}$ for 15 minutes, after which 250 
spectra were acquired, while recording the sample mount temperature using a PT1000 resistor. Temperature increments of $2 \mathrm{~K}$ (between $300 \mathrm{~K}$ and $312 \mathrm{~K}$ ) resulted in 1750 total recorded calibration spectra to provide the training data for the neural network.

Upon a successful calibration and alignment of the pump and probe beams, the position of the pump laser with respect to the probe laser was scanned between +2000 and $-2000 \mu \mathrm{m}$, with scanning steps of $50 \mu \mathrm{m}$. At each position, the laser was turned to full power $(1 \mathrm{~W})$ to provide enough modulated heating on the fiber to produce a sufficient photothermal signal. After a waiting period of 10 seconds (to allow the fiber to reach a stabilization of the gradual heat from the laser, resulting in mainly square wave modulated temperature oscillations), the spectrometer began measuring at a sampling rate of $50 \mathrm{~ms}$. The experiment ran sufficiently long enough to collect 100 periods of the excitation laser modulation, resulting in an improved phase measurement during the FFT analysis.

\section{Methods}

The following sections focus on: the neural network training process for time domain temperature reconstruction, the FFT procedure to find the amplitude and phase of the complex temperature (compared to the FFT of commonly used spectral features), development of a numerical method for generating the time evolution of the temperature dependent fluorescence spectra (assuming a linear relation between spectral features and temperature) at the modulation frequency, extending the numerical simulation to verify the ability of this method to extract the thermal diffusivity of the fiber from axial and frequency scans, and proof-of-concept of the method on experimental data.

\subsection{Neural Network Training and Temperature Recon- struction}

\subsubsection{Temperature Dependent Fluorescence of Spectra}

To reconstruct the temperature of the fiber surface, the relation between different spectral features and temperature (acquired during steady probe laser illumination but without pump laser-induced photothermal heating) was detailed. Several temperature dependent spectral features of the quantum dots (shape-factors) have been used independently in the literature and include: the peak intensity (PI) 28, the peak normalized intensity 29, the integrated intensity (II) 30, the PI/II ratio (R) 31, the peak wavelength (PW or PWL 32]), the FWHM [32], and the summed spectral bands 33. These features are shown as a function of temperature (along with the overall spectra) in Figure 2,

Being placed in a vacuum and maintaining all other variables to be constant, factors such as quenching, dipole interactions, $\mathrm{pH}$ [34], salt concentrations, and electron coupling 35] can be assumed not to have varied during the duration of the experiment, meaning that the spectral changes are solely functions of temperature. Furthermore, the fiber was illuminated for several hours under vacuum prior to the calibration procedure to avoid photobrightening and photodarkening [36]. The process to train the neural network based on these spectral features as inputs went as follows.

The neural network training process began with compiling the 250 spectra at each calibration temperature and then the intensity values every 10 wavelengths was averaged by a moving rectangular-window. This had the effect of reducing the noise induced by the spectrometer. A $3^{\text {rd }}$ order polynomial (for numerical stability) was then fit to the smoothed spectra, the derivative was taken, and the peak intensity and peak wavelengths were calculated at the maximum value. The integrated intensity was measured as the sum of the intensity values under the fit curve, and the ratio of peak intensity to integrated intensity was also calculated. The FWHM was also calculated based on the fitted curve. The "sum bands" consisted of the summed values of five $\approx 5 \mathrm{~nm}$ wide bands of the smoothed spectra between $536 \mathrm{~nm}$ and $664 \mathrm{~nm}$, similar to how photodiodes with notch filters would perform. Finally, the intensity values of 40 evenly spaced wavelengths of the spectra between $536 \mathrm{~nm}$ and $664 \mathrm{~nm}$ were normalized to the peak intensity and these values (in addition to the peak intensity) were ultimately selected as inputs for the neural network investigated in this work, based on providing the lowest RMS of the trained neural network $(0.29 \mathrm{~K})[12$.

\subsubsection{Neural Network Training}

Based on previous research and simulation [12, the neural network that provided the best accuracy was one where the inputs were based on 40 peak-normalized intensities and the peak intensity, containing 2 hidden nodes. The performance of this neural network is compared to the traditional use of peak intensity as a means to reconstruct the temperature in the time and frequency domains.

For cross-validation purposes, the temperature calibrated spectra were randomly distributed between training spectra (80\%) and testing spectra (20\%). The training data was used to vary the weights of the different nodes until the RMS error of the neural network output temperature compared to the expected temperature was minimized, guided by a Levenberg-Marquardt algorithm. The testing spectra were then used to confirm that the neural network was not over-trained. Figure 3 shows the general NN architecture, the expected vs output temperature of a trained neural network, and the averaged and unaveraged RMS error of the reconstructed temperature, based on experimental data. The results show that neural network is not over-trained and that the average RMS error from the calibration data is less than $0.05 \mathrm{~K}$ (Figure 3 ).

\subsection{Determination of Surface Temperature Oscillation Amplitude and Phase by FFT}

In order to map the spectral data signals from the time domain to the frequency domain, an FFT-based method 

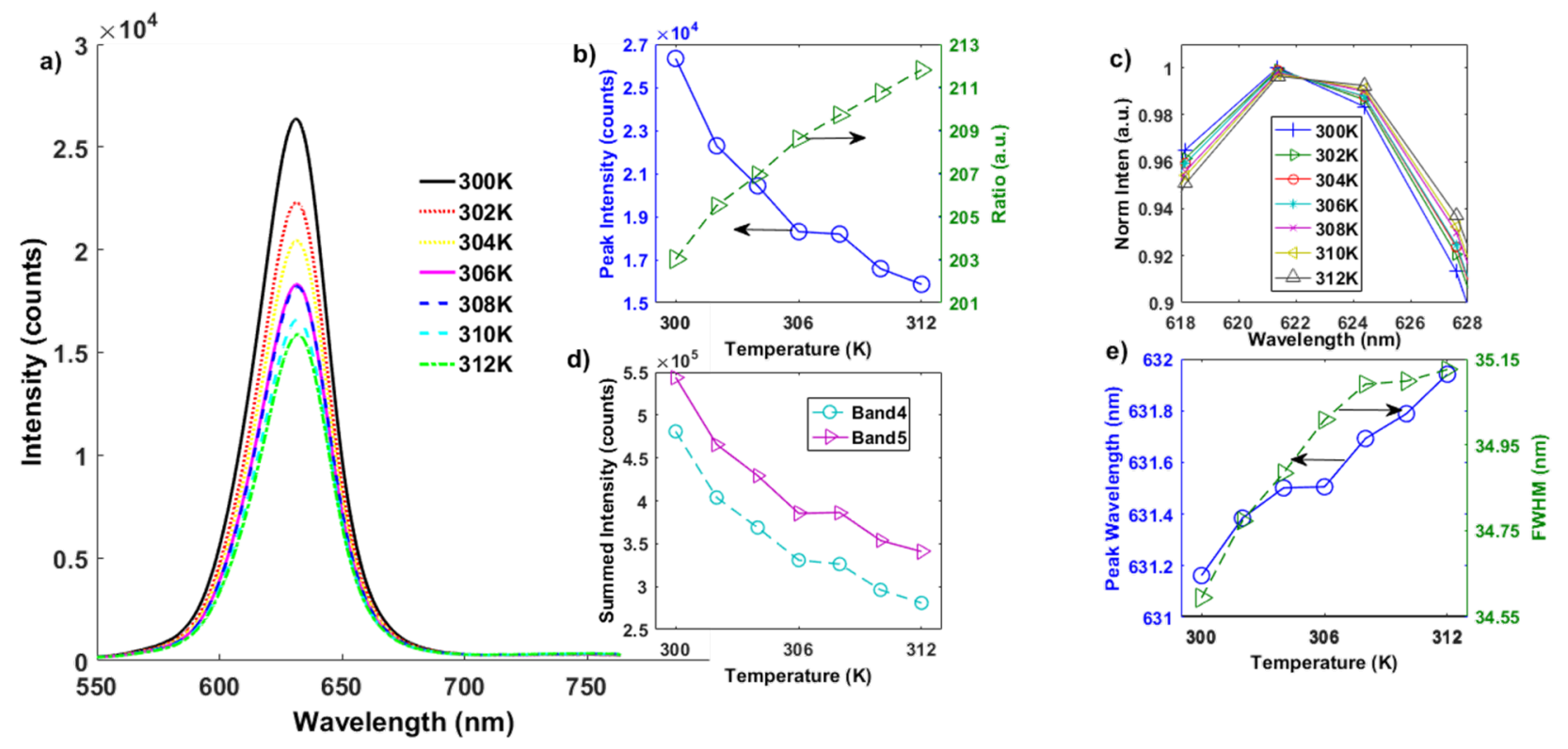

Figure 2: Fluorescent spectra of quantum dots from experimental results at the calibrated temperatures (a) and the temperature dependence of the Peak Intensity, Ratio (b), snapshot of 40 peak normalized intensities (c), snapshot of 2 of the 5 sum bands (d), and Peak Wavelength and FWHM (e) of the spectra.

was used. Residual green light from the modulated pump laser was sent into the spectrometer (because slight jitter in the optical chopper at the lower frequencies of the experiment prevented measuring the modulation behavior based on the embedded sensor in the chopper to get the proper modulation data), as well as the focused fluorescent emission. The reference signal of the experiment could be accurately monitored synchronously with the fluorescence spectra acquisition, by determining the intensity under the spectral peak magnitude at $532 \mathrm{~nm}$ for every acquired spectrum. An FFT was performed on the reference signal in the time domain, yielding the intensity modulation frequency, the modulation amplitude, and the modulation phase with respect to the chosen time scale. The modulation phase was stored to later be subtracted from the phase of the other signals. Furthermore, the phase of the modulated signal was used to determine the absolute phase delay between the extracted photothermal temperature modulation and the reference modulation.

An FFT was then performed on the NN-reconstructed temperature. Also the fluorescence peak intensity (PI) signal was analyzed via FFT, as it is a commonly used spectral feature in the literature to relate fluorescence to temperature. The peak intensity had the added benefit of a higher dynamic range (about $1 / 30000$ counts) than the NN-reconstructed temperature due to discretization effects of most of the other features (about $0.1 / 35 \mathrm{~nm}$ for FHWM and $0.1 / 631 \mathrm{~nm}$ for PWL) used to train some neural networks. To eliminate any DC offsets in the FFT signal, the time varying part of each signal was determined by fitting a $3^{\text {rd }}$ order polynomial to its time dependence signal, and subtracting the polynomial in order to remove effects of the slow temperature evolution due to the steady state component of the optical heating.

After performing the Fourier transform, the amplitude and phase of each signal (with respect to the phase of the reference signal) at the modulation frequency (as determined by the reference signal) was stored. The purpose of mapping the surface temperature in the frequency domain was to be able to use a frequency domain model for an infinitesimally small laser line heating source on a cylinder [15] and relate the acquired signal to the thermal diffusivity.

\subsection{Numerical Experiment for Assessing the NN Accu- racy and FFT Validity}

In order to verify the ability of the FFT-based method to accurately transform the spectral signals into the frequency domain for the purpose of measuring the thermal diffusivity of a thin fiber, a numerical simulation of the quantum dot spectra (approximated as a Gaussian distribution) and their behavior due to temperature variations was set up for different modulation frequencies and pumpprobe distances, mimicking the operation of a real life experiment and based on experimental uncertainties (Figure 4 and Table 1).

The evolution of the temperature as a function of time was simulated, with noise based on experimental data (Table 1) imposed on the temperature and temperaturedependent spectral features. Additional noise was added to the spectra to mimic the signal to noise ratio observed with the spectrometer in typical experimental conditions. The spectral features were assumed to be linear (based on experimental observations seen in Figure 2. 

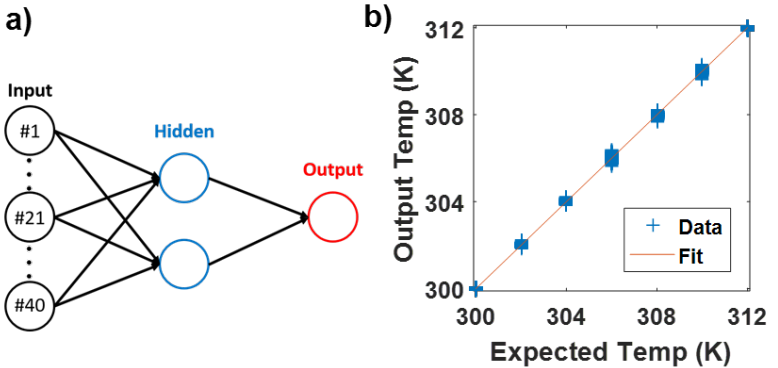

c)
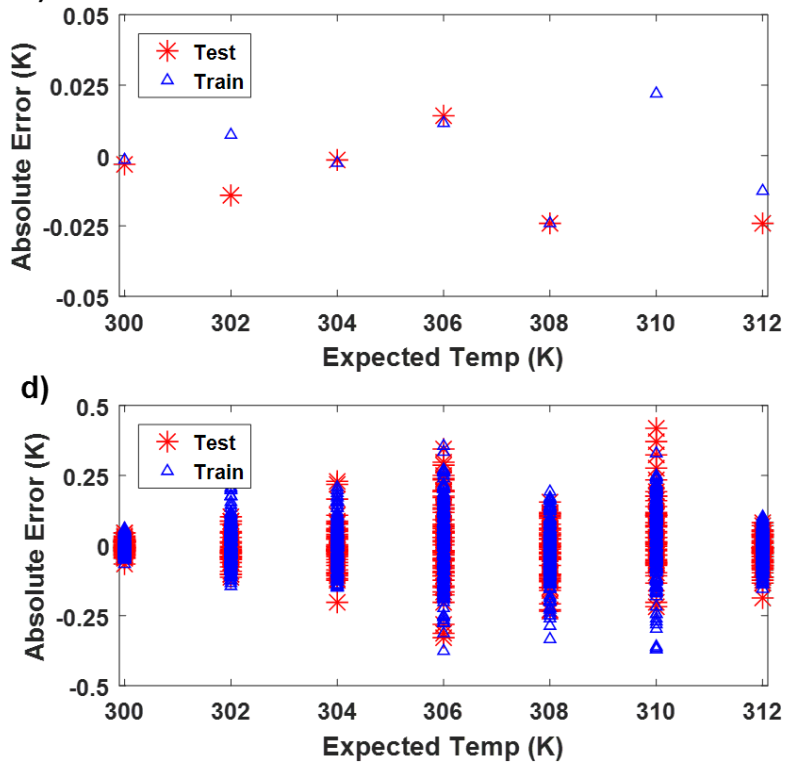

Figure 3: NN architecture for peak intensity and normalized intensities inputs (a), results of expected vs output temperature values from NN (b), and averaged RMS (c) and unaveraged (d) error values versus temperature from the NN training with training spectra results in blue triangles and test spectra in red stars. Experimental data.

Table 1: Inputs and uncertainties used for the simulation, based on experimental uncertainties.

\begin{tabular}{lrlrl}
\hline Sim. Parameters & Nominal Values & & Uncertainty & \\
\hline$\lambda$ Resolution & $520-770$ & $\mathrm{~nm}$ & 0.19 & $\mathrm{~nm}$ \\
PT1000 Temp & $300-312$ & $\mathrm{~K}$ & 0.05 & $\mathrm{~K}$ \\
Time Spacing & 0.001 & $\mathrm{sec}$ & 0.000727 & $\mathrm{sec}$ \\
Spectrometer Noise & 0 & counts & 217 & counts \\
$A_{P I, 0}$ & 329356 & counts & 42.58383 & counts \\
$B_{P W L, 0}$ & 613.51 & $\mathrm{~nm}$ & 0.123523 & $\mathrm{~nm}$ \\
$C_{F W H M, 0}$ & 16.153 & $\mathrm{~nm}$ & 0.000322 & $\mathrm{~nm}$ \\
$D_{0}$ & 1500 & counts & 50 & counts \\
$S_{P I}$ & -1014 & counts $/ \mathrm{K}$ & 1.391491 & counts $/ \mathrm{K}$ \\
$S_{P W L}$ & 0.059 & $\mathrm{~nm} / \mathrm{K}$ & 0.000404 & $\mathrm{~nm} / \mathrm{K}$ \\
$S_{F W H M}$ & 0.0616 & $\mathrm{~nm} / \mathrm{K}$ & 0.000105 & $\mathrm{~nm} / \mathrm{K}$ \\
$S_{D}$ & 0 & counts $/ \mathrm{K}$ & 0 & counts $/ \mathrm{K}$ \\
Motor Position & -2 to 2 and 0.5 & $\mathrm{~mm}$ & 0.0011 & $\mathrm{~mm}$ \\
$m_{\text {phase }}$ & 2000 & $\mathrm{rad} / \mathrm{m}$ & & \\
$m_{\text {mag }}$ & 600 & $1 / \mathrm{m}$ & & \\
$\alpha$ & 0.13 and 0.16 & $\mathrm{~mm}{ }^{2} / \mathrm{s}$ & & \\
$P_{0}$ & 1000 & $\mathrm{a} . \mathrm{u}$. & & \\
$k_{s}$ & 0.24 & $\mathrm{~W} / \mathrm{m} \mathrm{K}$ & & \\
$h$ & 6 & $\mathrm{~W} / \mathrm{m}^{2} \mathrm{~K}$ & & \\
$f$ & 0.5 and $2-10$ & $\mathrm{~Hz}$ & 0.05 & $\mathrm{~Hz}$
\end{tabular}
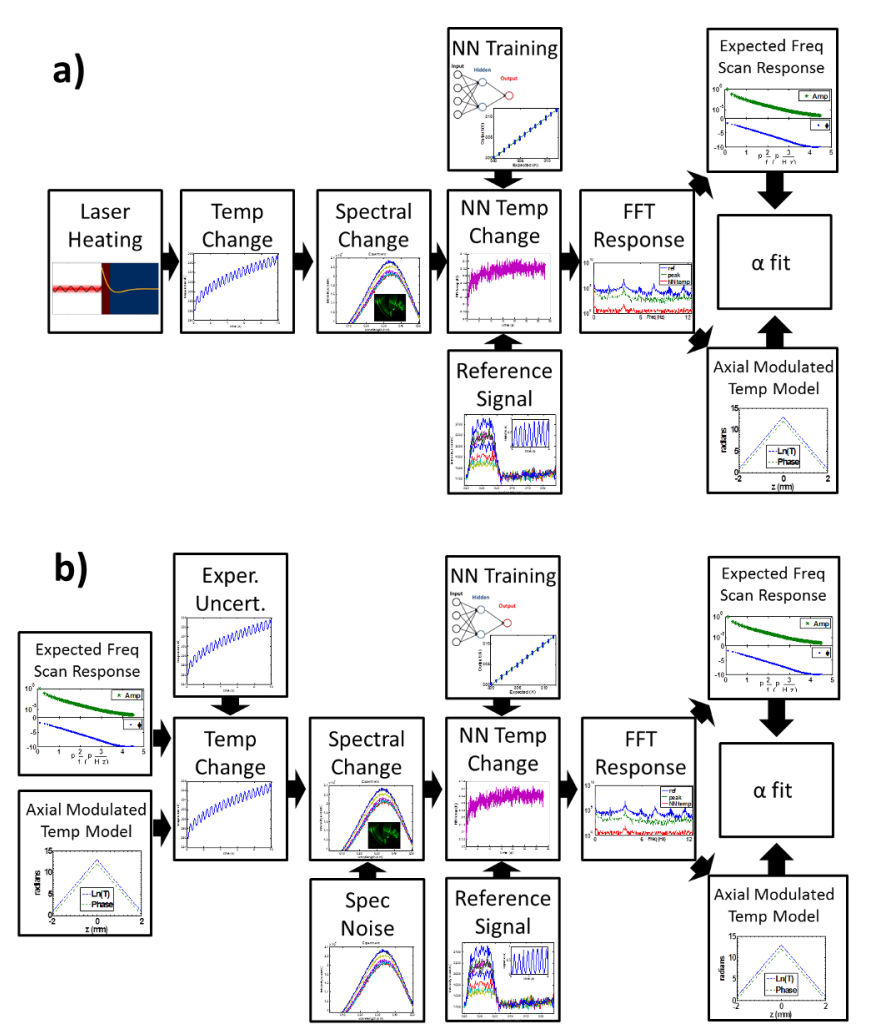

Figure 4: Illustration of the process to calculate the thermal diffusivity of a fiber, with respect to the photothermal signal amplitude and phase as a function of pump-probe spacing and laser(heating) intensity modulation frequency - experimental process (a) and numerical simulation (b). 
$\left(A=P_{P I, 0}+S_{P I} T\right)$ over the $300 \mathrm{~K}-312 \mathrm{~K}$ range of interest. In actuality, the different features become non-linear as the temperature range is increased, but the neural network is able to account for these non-linearities. The simulated spectra were then processed with the proposed FFT-based thermal property extraction method in the same way as the experiment, with the intent to compare the expected thermal properties to the calculated thermal properties by comparing the goodness of fit.

A model for heating of a cylinder by an intensity modulated, infinitesimally small laser line heating source was considered 15. The quantities of interest were the axial dependence of the amplitude and phase of the temperature oscillation on the fiber's surface, with the following conditions: sample suspended in vacuum to neglect convection, linearized radiation intensity, and the cylinder radius being significantly smaller than the diffusion length of the thermal wave. This provided the basis for modeling of the axial distribution of the fiber temperature at different modulation frequencies, Eqs. 1, 2, and 3.

$$
\begin{gathered}
\widetilde{T_{s}}(a, z) \approx \frac{P_{0}}{2 \pi k_{s} q_{s}^{\prime}} e^{-q_{s}^{\prime}|z|} \\
q^{\prime}=\sqrt{\frac{i \omega}{\alpha}+\frac{2 h}{k_{s} a}} \\
\mu=\sqrt{\frac{2 \alpha}{\omega}}
\end{gathered}
$$

The model predicts a linear dependence on the pumpprobe distance of the phase delay and of the logarithm of the magnitude when the heated region is infinitesimally narrow, Eqs. 4 and 5

$$
\begin{gathered}
z_{\text {mag }}=\exp \left(m_{\text {mag }}|z|+b_{\text {mag }}\right) \\
z_{\text {phase }}=m_{\text {phase }}|z|+b_{\text {phase }}
\end{gathered}
$$

The slopes of the phase and natural log of the magnitude are determined by the modulation frequency and the fiber's thermal diffusivity, Eq. 6.

$$
m_{\text {phase }} \times m_{\text {mag }}=-\pi f \alpha^{-1}
$$

Two types of scans were numerically investigated, a frequency scan at a fixed axial pump-probe spacing of $0.5 \mathrm{~mm}$ and an axial scan at a fixed frequency of $0.5 \mathrm{~Hz}$. During the axial scan, the frequency was fixed at $0.5 \mathrm{~Hz}$, resulting in values for the phase and magnitude slopes (Eqs. 4 and 5 of $2000 \mathrm{rad} / \mathrm{m}^{-1}$ and $500 \mathrm{~m}^{-1}$, respectively, for a chosen diffusivity $(\alpha)$ value of $1.3 \times 10^{-7} \mathrm{~m}^{2} \mathrm{~s}^{-1}$ (Eq. 6).

To mimic the time domain behavior of the experiment, the temperature oscillations (indicative of the complex temperature), Eq. 77 were superimposed onto a gradual temperature rise (Eq. 8), with a power law shape with exponent $1 / 2$, which was indicative of the sample heating that occurred from the DC offset of the laser heating.

$$
\begin{aligned}
& T_{A C}(z \text { or } f, t)=\frac{f r e q_{\text {mag }}}{f r e q_{\text {mag }, \text { max }}} \frac{z_{\text {mag }}}{z_{\text {mag }, \text { max }}} \\
& \times \cos \left[2 \pi f\left(t-t_{0}\right)-z_{\text {phase }}-f r e q_{\text {phase }}\right] \\
& T_{D C}(z \text { or } f, t)=\left[T_{\text {end }}-T_{0}\right]\left(\frac{t-t_{0}}{t_{\text {end }}}\right)^{n}+T_{0}
\end{aligned}
$$

The linearly dependent spectral features $\left(A_{P I, 0}, S_{P I}\right.$, $B_{P W L}, S_{P W L}$, etc), with values measured from experiment (Table 1), were used to create Gaussian-shaped spectra changing with time, Eq. 9. The green reference signal from the modulated laser was simulated as a trapezoidal curve between $526 \mathrm{~nm}$ and $535 \mathrm{~nm}$ and was added to the simulation at the heating modulation frequency.

$$
\begin{aligned}
\operatorname{Spec}[ & \lambda, T(z \text { or } f, t)]=\left(A_{P I, 0}+S_{P I} T\right) \\
& \times \exp \left[\frac{-4 \ln (2)\left(\lambda-\left(B_{P W L, 0}+S_{P W L} T\right)\right)^{2}}{\left(C_{F W H M, 0}+S_{F W H M} T\right)^{2}}\right]
\end{aligned}
$$

The simulation created calibration spectra for the training of the neural network (steady temperatures), in addition to the modulated spectra. Experimental uncertainties (Table 1) were used in the generation of both types of spectra.

\section{Results}

\subsection{FFT Behavior via Simulation}

Using the simulated data from the previous section (where the nominal values and uncertainty in the inputs were based on experimental data), the data processing described in Figure 4 was extended to the reconstruction of photothermally induced temperature evolutions. As mentioned above, an FFT was performed on the reference signal to find the modulation frequency. Figure 5 shows the simulated results of the amplitude of the FFT for a simple sinusoidal modulation. A peak at $0.5 \mathrm{~Hz}$ is observed in the reference signal and corresponding peaks are seen in the reconstructed NN temperature and the peak intensity (PI) of the signal.

The results in Figure 5 demonstrate that mapping the fluorescent spectral features signal into the frequency domain via FFT can provide the amplitude and phase at the modulation frequency, and that the modulation frequency can be determined by the FFT of the reference signal.

Additionally, the windowing of the entire signal was selected to observe the effect of including the curved baseline (caused by DC portion of heating) compared to subtracting a $3^{\text {rd }}$ order polynomial fit from the signal prior to implementation of the FFT. The subtracted signal provides the lowest error on the phase of the signal, and still maintains the high amplitude of the signal. Compared to subtracting the baseline, an error of $1.1 \%$ on the amplitude 


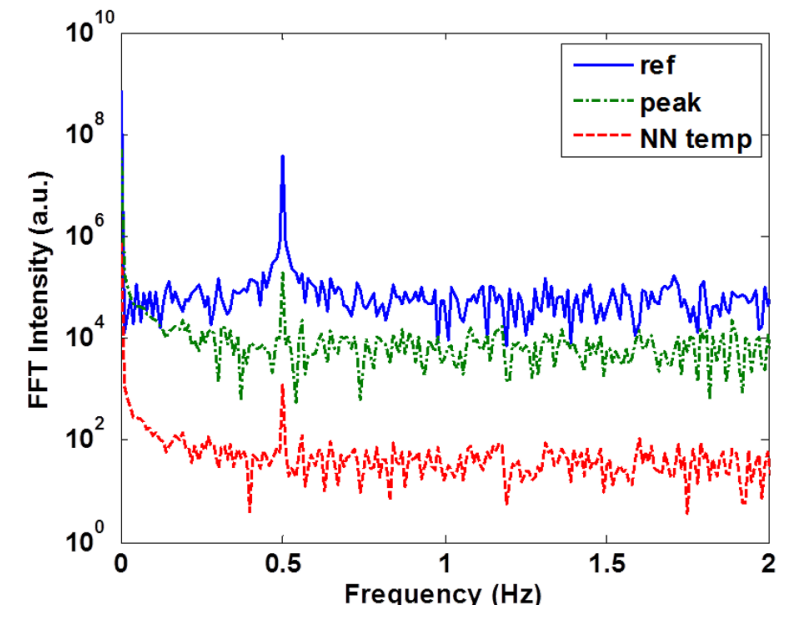

Figure 5: FFT spectra for numerical simulation (sine modulation at $0.5 \mathrm{~Hz}$ ) showing the dominant frequency present in the reference with corresponding FFT peaks in the spectral signals (NN temperature and Peak Intensity) at the modulation frequency of the original temperature.

of the signal is observed due to the gradual temperature rise. The effect on the phase (compared to the true phase) is $1.24^{\circ}$ without and $0.11^{\circ}$ with the $3^{\text {rd }}$ order polynomial subtraction. The results presented in this work are based on FFT values after the polynomial subtraction.

\subsection{Frequency Scan and Axial Scan Simulation}

In the following, we verify if the dependences of the reconstructed temperature oscillation amplitude and phase on the modulation frequency and pump-probe distance (as calculated by simulated spectra) are well reconstructed. For the numerical simulations, the normalized intensity neural network was selected to analyze the experimental behavior of photothermally heating a fiber.

As explained in section 3.3, the model derived by Salazar et al. 15] was used for a simulated frequency scan of the temperature. The phase and amplitude of 81 linearly spaced frequencies between $2 \mathrm{~Hz}$ and $10 \mathrm{~Hz}$ were determined at a fixed axial spacing of $0.5 \mathrm{~mm}$ (Eqs. 1 and 2 ) and then used as inputs in Eq. 7) where the amplitude was normalized to the maximum value observed at the lowest frequency. The spectra, calculated as explained in section 3.3. were then presented to the neural networks [37] to reconstruct the surface temperature.

For each scanning frequency, the FFT method described above was applied to the reference signal, the NN reconstructed temperature, and the peak intensity signal. The resulting frequency dependence of the amplitude and phase of the photothermal signal was compared to the reference signal (modulated temperature) underlying the simulation, to verify the performance of the reconstruction procedure. The results of the frequency scan (Figure 6 a and b) show good agreement at low frequencies between the frequency dependence of the temperature used to create the NN inputs, the peak intenisty signal, and the
NN-reconstructed data. However, at higher frequencies, the NN-reconstructed signal at the modulation frequency is buried in the noise and signal decay with frequency saturates at the noise floor (Figure 6r). Table 1 gives these noise values, which results in a noise level of about $-14 \mathrm{~dB}$ ) corresponding to a modulated temperature of near 0.07 $\mathrm{K}$ ) on the peak intensity and the noise floor observed in the frequency scan is about $-13 \mathrm{~dB}$.

The simulation was also run to mimic an axial scan, with the input amplitude and phase data calculated by Eqs. 1 and 22, at a frequency of $0.5 \mathrm{~Hz}$. The two-sided results (Figure 7) of the output phase and amplitude compared to the input agree well. One thing of note during operation of the simulation was that the preferred range of frequencies (to avoid significant attenuation of the signal, while still operating within the acceptable range of the spectrometer) was between $0.5 \mathrm{~Hz}$ to $10 \mathrm{~Hz}$ for a frequency scan, and near $0.5 \mathrm{~Hz}$ for an axial scan. However, the method is severely limited by the level of noise that exists in the reference signal (i.e. $-10 \mathrm{~dB}$ of noise superimposed on the reference signal makes it difficult to correctly identify the modulation peak in the reference signal) to get an accurate reconstruction of the phase delay. Further work is to be done on improving the reference signal for improved phase detection.

\subsection{Experimental Results}

The application of the neural network temperature determination thus far described was also extended to the experiment described in section 2, The experimental setup that made use of an optical chopper and recorded by spectrometer could not provide a sufficiently large range of frequencies to be tested for use in a frequency scan (minimum allowable frequency $\approx 1.8 \mathrm{~Hz}$, limited by optical chopper; maximum allowable frequency $\approx 5 \mathrm{~Hz}$, limited by integration time of spectrometer to acquire sufficient signal). Because of that limitation, only an axial scan at fixed frequency was performed to deliver a proof-of-concept for the method.

The decay with pump-probe distance of the amplitude of the photothermal signal as reconstructed from the time dependent fluorescence spectra at different pump-probe distances is presented in Figure 8a. The scanning step was 50 $\mu \mathrm{m}$, with a scanning distance of $-2 \mathrm{~mm}$ to $2 \mathrm{~mm}$ spacing between the pump and probe beams. The sampling frequency of the spectrometer was $13.8 \mathrm{~Hz}$ and an average of 825 spectra were acquired per axial position. To improve the accuracy of the FFT of the signal, the slow temperature rise was removed by fitting of a $3^{\text {rd }}$ order polynomial to the signal and then subtracted, so that the AC-portion of the signal remained. This was obtained via the same FFT process described previously, including selecting only the values of the FFT signal at the fundamental frequency (due to square wave modulation from the optical chopper).

Figure $8 \mathrm{a}$, shows the modulated behavior of the fluorescence peak intensity signal based on the experimental reference signal (based on both the green light and some 

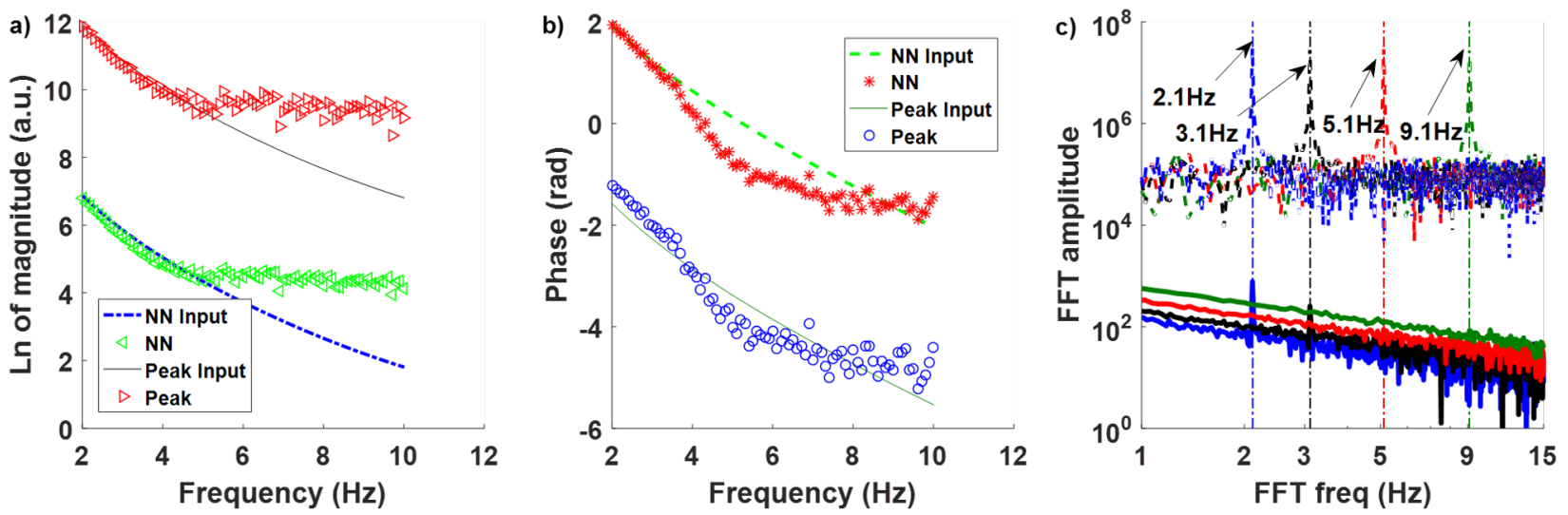

Figure 6: Simulated results, amplitude (a) and phase (b), of FFT-based method for frequency scan with respect to the NN reconstructed temperature and peak intensity signals. The reconstruction approach is effective in reconstructing the input amplitude and phase lower frequencies, but is limited due to the signal being buried in the noise at higher frequencies (c).

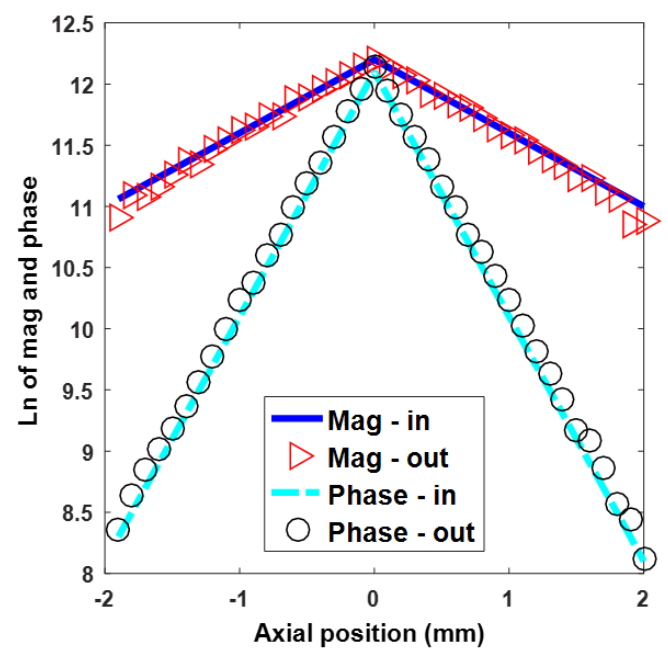

Figure 7: Simulated results of the FFT-based NN method for an axial scan of a photothermally generated temperature field. The reconstruction method is correctly determining the amplitude and phase of the modulated signals. residual IR light that entered the spectrometer). The values were normalized to their peak values and show, as expected, that when the pump laser was on (high reference signal), the fluorescence peak intensity decreased due to the increase in temperature from the laser heating. Figure $8 \mathrm{p}$, shows the FFT spectra of the experimental square wave modulation reference, the NN reconstructed temperature, and the peak intensity signals, with higher harmonic elements observable.

A broad central peak reflecting the finite size of the excitation spot and of the probed area was observed during the axial scan of the pump-probe distance. The absolute phase delay of the reference signal phase subtracted from the temperature signal phase was erratic at the different axial positions due to too much noise in the reference signal. This noisy reference signal is the limiting factor in accurately applying this method to determine thermal properties, as explained in the end of section 4.2. Additionally, the numerical simulations for the axial thermal wave decay had assumed an infinitely narrow laser beam and no effect from the background at low signal noise levels. However, in practice this is not valid and therefore, the more complete model by Salazar [15] must be used and are presented in Eq. 10

$$
\begin{aligned}
\widetilde{T_{s}}(a, z) \approx & \int_{-\infty}^{\infty} e^{i \lambda z} \frac{P_{0}}{4 \pi k_{s} \delta_{s}} \times \\
& \frac{e^{-\lambda^{2} b^{2} / 8}}{\frac{\delta_{s} a}{2}-\frac{k_{g} \delta_{g}}{k_{s} \delta_{s}} \frac{1}{\delta_{g} a \ln \left(\delta_{g} a\right)}+\frac{h}{k_{s} \delta_{s}}} \mathrm{~d} \lambda,
\end{aligned}
$$

where $\delta$ is defined as $\delta_{i}^{2}=q_{i}^{2}+\lambda^{2}$ and $q_{i}=\sqrt{i \omega / D}$.

The data presented in Figure 8 a, where only data above the noise floor were selected, was used to curve fit the magnitude of Eq. 10, with the thermal diffusivity and beam width being the two curve fitting parameters. The 
a)

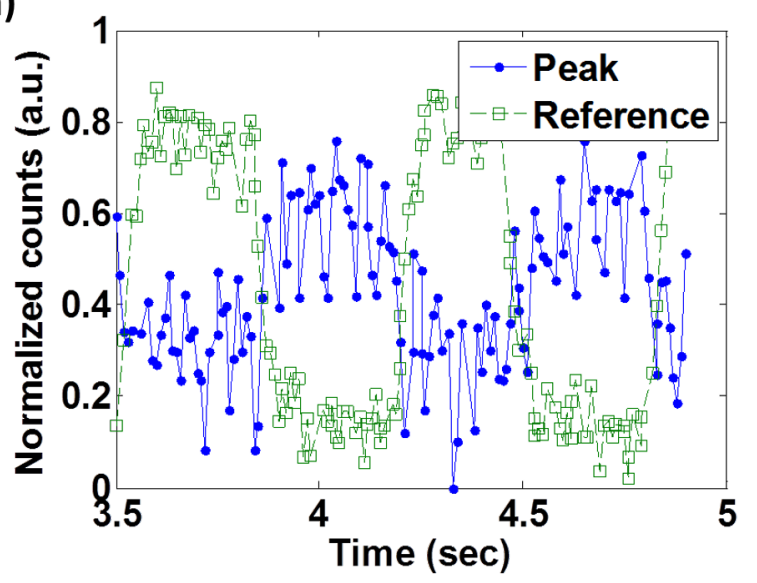

b) 10

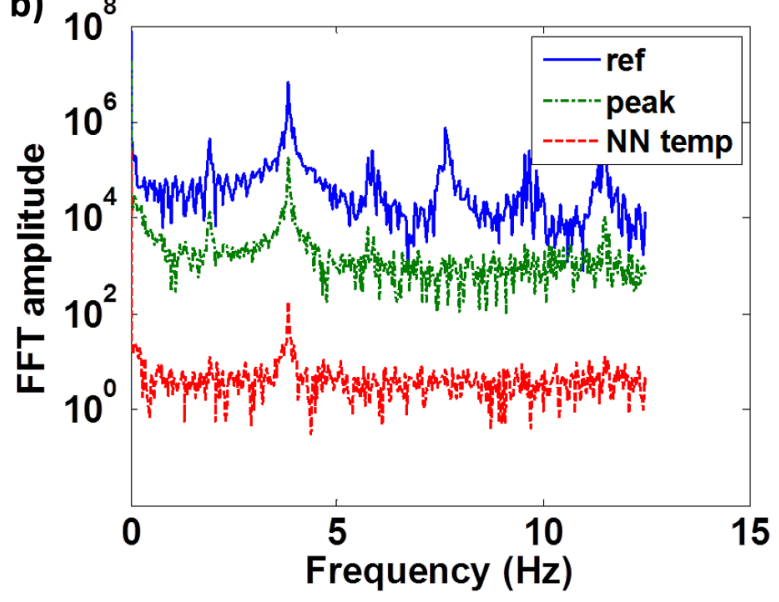

Figure 8: The modulated behavior of the reference and temperature dependent signals (a). The FFT spectra at a chopper modulation frequency of $2 \mathrm{~Hz}$ at one of the axial positions (b) showing dominant frequencies present in the reference with corresponding FFT peaks in the spectral signals (NN temperature and Peak Intensity) at the fundamental frequency clearly. Because of the uneven duty cycle of the $2 \mathrm{~Hz}$ square wave modulation via optical chopper, higher frequency components of the reference signal are observed at all harmonics of the $2 \mathrm{~Hz}$ signal, while only the $4 \mathrm{~Hz}$ peak is observed in the NN temperature and the $2 \mathrm{~Hz}, 4 \mathrm{~Hz}$, and $12 \mathrm{~Hz}$ peaks are observed in the peak intensity. a)

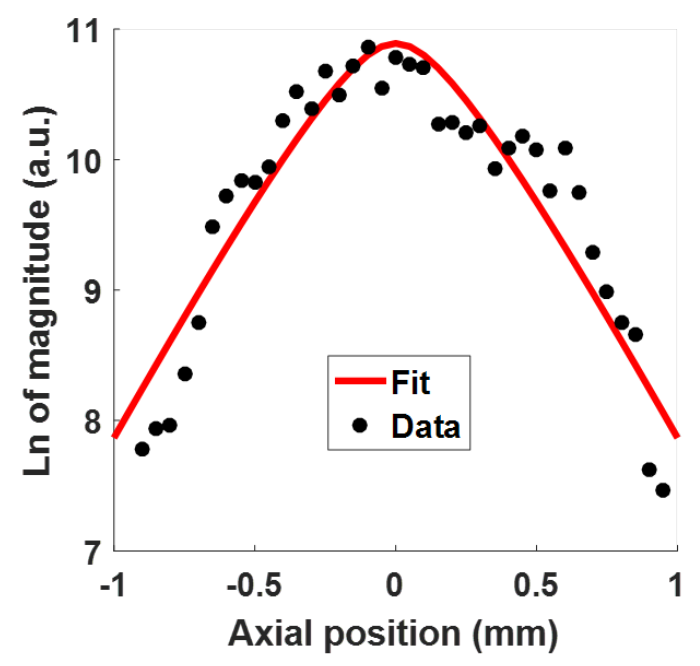

b)

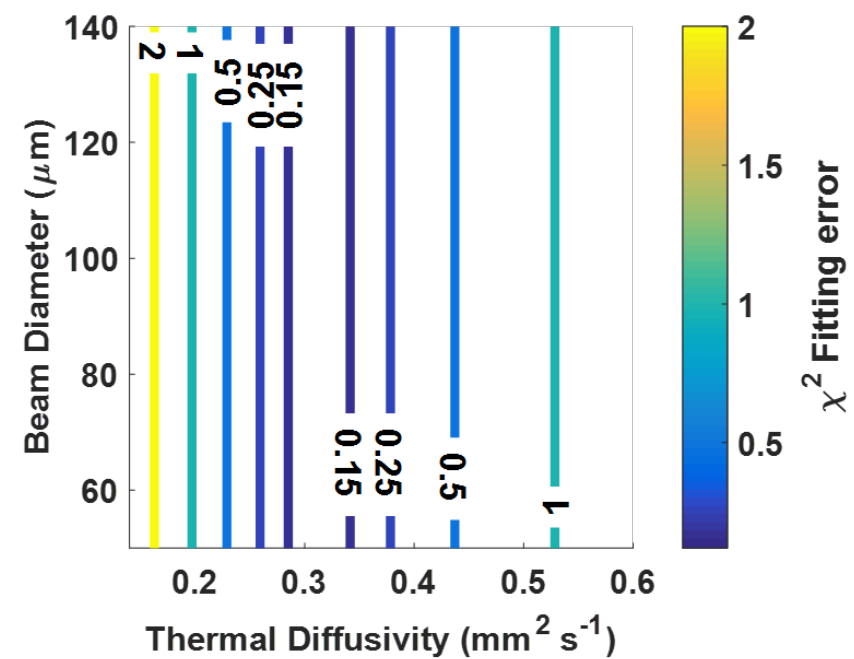

Figure 9: Curve fit of magnitude of peak fluorescent intensity (a) with data boints below the noise floor ignored. Fitting error (b) based on beam width and fiber thermal diffusivity, showing the insensitivity to beam diameter on the fitting. 
selection of beam width being a fitting parameter was because of the uncertainty in the degree of overlap between the IR pump beam (about $90 \mu \mathrm{m}$ in diameter) and the green probe beam to induce fluorescence (about $30 \mu \mathrm{m}$ in diameter). The results of the curve fit are presented in Figure 9, with the measured thermal diffusivity based on amplitude alone being $3 \pm 0.8 \times 10^{-7} \mathrm{~m}^{2} \mathrm{~s}^{-1}$ and the fitting beam width being $\approx 110 \mu \mathrm{m}$, which is near the quadratically summed diameter of the two beams. This diffusivity value is comparable to some of the more recent synthetic silks as measured by the Transient Electrothermal technique (TET) [24] $\left(3.3 \pm 0.8 \times 10^{-7} \mathrm{~m}^{2} \mathrm{~s}^{-1}\right)$, which demonstrates the proof-of-concept of the proposed method. Additionally, the current method requires significantly less time to implement and take a measurement than does the TET technique. Similar to the frequency scan in Figure 6, the modulated temperature signal becomes buried in the noise at about a $1 \mathrm{~mm}$ axial spacing between the lasers, with the noise floor being about -15 $\mathrm{dB}$, or about $0.05 \mathrm{~K}$.

\section{Conclusions}

$\mathrm{CdSe} / \mathrm{ZnS}$ quantum dot shape-based thermometry via neural network has been shown to serve as a potential basis for an FFT-based detection of photothermally induced temperature variations, while maintaining absolute temperature information in the time domain from the neural network. A numerical simulation reconstructing the frequency and pump-probe distance dependence of a photothermal signal from fluorescence spectra demonstrated the intrinsic feasibility of the approach for thermal property determination. Experimental results for an axial scan qualitatively confirm that the amplitude of the signal decays with pump-probe distance in an expected manner, resulting in a thermal diffusivity measurement for a synthetic spider silk fiber of $3 \pm 0.8 \times 10^{-7} \mathrm{~m}^{2} \mathrm{~s}^{-1}$. Methods to improve measurement of the phase will be investigated in a future article and will aid in the determination of the thermal diffusivity of thin fibers, as well as improvements in temperature reconstruction accuracy to lower the noise floor.

\section{Acknowledgments}

The authors would like to thank the members of the Multiscale Thermalphysics Lab and the Soft Matter and Biophysics Lab. L. Liu acknowledges the support of the Chinese Scholarship Council (CSC).

\section{References}

[1] G. W. Walker, V. C. Sundar, C. M. Rudzinski, A. W. Wun, M. G. Bawendi, and D. G. Nocera, Applied Physics Letters 83, 3555 (2003).

[2] U. Resch-Genger, M. Grabolle, S. Cavaliere-Jaricot, R. Nitschke, and T. Nann, Nature Methods 5, 763 (2008).
[3] W. G. van Sark, P. L. Frederix, D. J. Van den Heuvel, H. C. Gerritsen, A. A. Bol, J. N. van Lingen, C. de Mello Donega, and A. Meijerink, The Journal of Physical Chemistry B 105, 8281 (2001).

[4] G. Kucsko, P. Maurer, N. Yao, M. Kubo, H. Noh, P. Lo, H. Park, and M. Lukin, Nature 500, 54 (2013).

[5] T. Jamieson, R. Bakhshi, D. Petrova, R. Pocock, M. Imani, and A. M. Seifalian, Biomaterials 28, 4717 (2007).

[6] S. Li, K. Zhang, J.-M. Yang, L. Lin, and H. Yang, Nano Letters 7, 3102 (2007).

[7] D. Jaque, B. d. Rosal, E. M. Rodríguez, L. M. Maestro, P. HaroGonzález, and J. G. Solé, Nanomedicine 9, 1047 (2014).

[8] B. Han, W. L. Hanson, K. Bensalah, A. Tuncel, J. M. Stern, and J. A. Cadeddu, Annals of Biomedical Engineering 37, 1230 (2009).

[9] V. Pilla, E. Munin, N. O. Dantas, A. C. Silva, A. A. Andrade, and A. Al-Ahmadi, Quantum Dots-A Variety of New Applications , 3 (2012).

[10] L. Liu, S. Creten, Y. Firdaus, J. J. A. F. Cuautle, M. Kouyaté, M. Van der Auweraer, and C. Glorieux, Applied Physics Letters 104, 031902 (2014).

[11] E. V. Shashkov, M. Everts, E. I. Galanzha, and V. P. Zharov, Nano Letters 8, 3953 (2008).

[12] T. Munro, L. Liu, C. Glorieux, and H. Ban, Journal of Applied Physics 119, 214903 (2016), http://dx.doi.org/10.1063/1.4953223

[13] J. R. Lakowicz, Principles of fluorescence spectroscopy (Springer Science \& Business Media, 2013).

[14] D.-s. Wang, G.-m. Wang, W.-w. Pan, and Y.-t. Wang, Optoelectronics Letters 4, 147 (2008).

[15] A. Salazar, A. Mendioroz, R. Celorrio, et al., Journal of Applied Physics 107, 043508 (2010).

[16] Z. Hua, H. Ban, and D. H. Hurley, Review of Scientific Instruments 86, 054901 (2015).

[17] S. Allison and G. Gillies, Review of Scientific Instruments 68, 2615 (1997).

[18] J. Ravi, Y. Lu, S. Longuemart, S. Paoloni, H. Pfeiffer, J. Thoen, and C. Glorieux, Journal of Applied Physics 97, 014701 (2005).

[19] R. Fuente, A. Mendioroz, and A. Salazar, Materials Letters 114, 1 (2014).

[20] X. Huang, G. Liu, and X. Wang, Advanced Materials 24, 1482 (2012).

[21] C. Xing, T. Munro, B. White, H. Ban, C. Copeland, and R. Lewis, Polymer 55, 4226 (2014).

[22] S. Shen, A. Henry, J. Tong, R. Zheng, and G. Chen, Nature Nanotechnology 5, 251 (2010).

[23] V. Singh, T. L. Bougher, A. Weathers, Y. Cai, K. Bi, M. T. Pettes, S. A. McMenamin, W. Lv, D. P. Resler, T. R. Gattuso, et al., Nature Nanotechnology 9, 384 (2014).

[24] C. Xing, T. Munro, C. Jensen, and H. Ban, Measurement Science and Technology 24, 105603 (2013).

[25] R. Dacal, Thermal and Optical Characterization of Heterogeneous Materials by Infrared Lock-in Thermography, Ph.D. thesis, UPV/EHU (2012).

[26] D. C. Prevorsek, Journal of Polymer Science Part C: Polymer Letters 26, 326 (1988).

[27] C.-H. Kwan, A. Matvienko, and A. Mandelis, Review of Scientific Instruments 78, 104902 (2007).

[28] N. Vyhnan and Y. Khalavka, Luminescence (2013), 10.1002/bio.2600

[29] J. Arigita, B. Larrion, J. Bravo, M. Hernaez, I. R. Matias, and F. J. Arregui, in Sensors, 2008 IEEE (IEEE, 2008) pp. 351-354.

[30] J. Bravo, J. Goicoechea, J. M. Corres, F. J. Arregui, and I. R. Matias, in Third European Workshop on Optical Fibre Sensors (International Society for Optics and Photonics, 2007) pp. 661919-661919.

[31] S. Ebert, K. Travis, B. Lincoln, and J. Guck, Opt. Express 15, 15493 (2007).

[32] S. F. Wuister, C. de Mello Donegá, and A. Meijerink, Journal of the American Chemical Society 126, 10397 (2004).

[33] L. Liu, K. Zhong, T. Munro, S. Alvarado, R. Côte, S. Creten, 
E. Fron, H. Ban, M. Van der Auweraer, N. Roozen, et al., Journal of Applied Physics 118, 184906 (2015).

[34] D. Aigner, S. M. Borisov, F. J. O. Fernández, J. F. F. Sánchez, R. Saf, and I. Klimant, Talanta 99, 194 (2012).

[35] M. Sauer, J. Hofkens, and J. Enderlein, Handbook of Fluorescence Spectroscopy and Imaging: From Single Molecules to Ensembles , 1 (2011).

[36] J. J. Peterson and T. D. Krauss, Physical Chemistry Chemical Physics 8, 3851 (2006).

[37] C. Glorieux and J. Thoen, Journal of Applied Physics 80, 6510 (1996). 